\title{
Philosophiques
}

\section{Le fédéralisme, l'État et la démocratie}

\section{Vincent di Norcia}

Volume 8, numéro 1, avril 1981

URI : https://id.erudit.org/iderudit/203156ar

DOI : https://doi.org/10.7202/203156ar

Aller au sommaire du numéro

Éditeur(s)

Société de philosophie du Québec

ISSN

0316-2923 (imprimé)

1492-1391 (numérique)

Découvrir la revue

Citer ce document

di Norcia, V. (1981). Le fédéralisme, l'État et la démocratie. Philosophiques, 8(1), 167-184. https://doi.org/10.7202/203156ar d'utilisation que vous pouvez consulter en ligne.

https://apropos.erudit.org/fr/usagers/politique-dutilisation/ 


\title{
LE FÉDÉRALISME, L'ÉTAT, ET LA DÉMOCRATIE
}

\author{
par Vincent di Norcia*
}

«Grandeur des nations, étendue des États; première et principale source des malheurs du genre humain.»

Jean-Jacques Rousseau'.

$\mathrm{La}$ "première et principale source des malheurs» du fédéralisme canadien réside à mon avis dans sa politique de grandeur étatiste ${ }^{1}$. Et j'ignorerai volontiers la question "du genre humain». L'étatisme est la tendance qu'ont les systèmes politiques à devenir indépendants par rapport à la volonté populaire. Cette tendance se trouve aujourd'hui renforcée non pas tant par la dictature que par la pure complexité de l'appareil d'État et par l'autocroissance de cet appareil. Contre l'étatisme, je propose l'accroissement de la démocratie, la maximisation des formes démocratiques d'autonomie inhérentes à notre culture politique. Ceci m'amène à proposer une reconstruction démocratique du fédéralisme canadien sur la toile de fond d'un pacte social entre les communautés qui composent le Canada ${ }^{2}$.

\section{UN FÉDÉRALISME ÉTATISTE}

L'État exécutif fort fait partie de la tradition canadienne. Le système canadien constitue ainsi une union fortement centralisée mais très peu fédérale. Chaque gouvernement membre, central

* Ce texte a été traduit de l'anglais par mon collègue, le professeur André Mineau.

1. Dans COBBAN, A., Rousseau and the Modern State, London, 1964, 121; au sujet de la démocratie, voir C.B. Macpherson, The Life and Times of Liberal Democracy, Toronto, 1977.

2. Voir d'ENTREVES, A.-P., The Notion of the State, 1967; et BALANDIER, G., Political Antbropology, New York, 1970, 6e chap. 
ou provincial, constitue lui-même un État. À chaque niveau de gouvernement, ce sont la Couronne et le Parlement (incluant les Chambres hautes dont les membres sont nommés) qui sont constitutionnellement souverains, et non le peuple.

L'autonomie des représentants élus est renforcée par la synthèse des fonctions exécutives et législatives, et par la prédominance du Cabinet qu'engendre cette synthèse. De plus, la prédominance de la bureaucratie d'État renforce l'autonomie de chaque niveau de gouvernement par rapport au contrôle populaire.

Cette autonomie dans l'exercice du pouvoir a été mise en relief par la manière dont les colonies britanniques ont décidé de leur union. L'Union de 1840 était unilingue anglaise, ne tenait pas compte de la majorité canadienne-française, et n'avait pas de gouvernement responsable face au Parlement, en dépit des demandes populaires de l'époque. La création d'une union coloniale plus grande, en 1867, a été une réponse aux initiatives des élites coloniales gouvernantes et des officiers impériaux, beaucoup plus qu'aux requêtes populaires. Elle n'a été populaire qu'en Ontario, tandis qu'elle a rencontré de vives résistances dans toutes les autres colonies. Le processus décisionnel impliqua seulement les élites gouvernantes des colonies d'abord, et ensuite celles de l'Empire. On ne se préoccupa ni de l'opinion ni de l'accord du peuple. Même les Législatures furent ignorées ${ }^{3}$.

La forme de l'Union elle-même était plus étatiste et centralisée que fédérale, ainsi qu'on l'admettait ouvertement à l'époque. Le fédéralisme était alors défini en termes de composition du Sénat et, à un degré moindre, de garanties diverses accordées au Québec. L'Union n'était tout simplement pas une "Confédération». L'interprétation moderne du fédéralisme en termes de souveraineté divisée est étatiste plutôt que démocratique, tout comme la souveraineté en question.

On a ouvertement donné à l'État central tous les principaux pouvoirs du gouvernement. Cette division des pouvoirs reflétait d'ailleurs celle qui prévalait entre l'Empire et les colonies avant la création de l'Union. Cette relation impériale fut renforcée par le

3. Cf. BLACK, E., Divided Loyalties, Montréal, 1975; HOCKIN, T., Governement in Canada, Toronto, 1976, 2e ch.; FRENCH, S., ed., La Confédération canadienne: qu'en pensent les philosophes?, Montreal, 1979, I et II; et WAITE, P.B., The Life and Times of Confederation, Toronto, 1962. 
pouvoir résiduel de l'État central, par ses pouvoirs de nommer les lieutenants-gouverneurs, les juges des cours les plus élevées et les sénateurs, de déclarer tout travail public et national, de passer par-dessus les provinces en ce qui concerne les écoles confessionnelles, d'homogénéiser le droit civil à l'extérieur du Québec, et, finalement de rejeter toute loi provinciale ${ }^{4}$. L'Union est donc plus étatiste que fédérale.

L'étatisme, en pratique, est la tendance qu'ont les États à étendre leur territoire et à accroître leurs pouvoirs. Cette tendance apparaissait déjà clairement à Christopher Dunkin, un député du Québec, qui, en 1865 , avait critiqué d'une manière prophétique l'aspect grandiose et coûteux de l'État canadien, de même que sa confusion des juridictions. Dans l'espace d'une génération, la tendance en question a engendré des conflits et de la concurrence entre les États provinciaux et l'État central. À mesure que les économies provinciales se sont modernisées, en Ontario d'abord il y a 80 ans, et, plus récemment, au Québec et en Alberta, les gouvernements de ces provinces ont réagi contre la domination du système par l'État central. Certains conflits intergouvernementaux sont devenus si profonds que les relations entre les gouvernements concernés sont devenues comparables à des négociations diplomatiques internationales entre États souverains séparés ${ }^{5}$.

C'est dans cette perspective de compétition intergouvernementale qu'il faut voir les demandes des provinces pour une plus grande autonomie, et leurs attaques contre l'État central. Elles ont débuté dès 1887, en Ontario. Elles ont été renforcées par le parti pris provincialiste des interprétations constitutionnelles littérales, souvent tortuées, du Conseil privé britannique au cours des 50 premières années de l'Union. Les débats économiques sur la question du laissez-faire et de l'intervention de l'État ont été transformés en compétition intergouvernementale pour l'exercice des principaux pouvoirs économiques ${ }^{6}$.

4. Voir l'Acte de l'A mérique du Nord britannique, articles 9,56 à 60, 91, 92.10c, 93 à 96; et KENNEDY, W.L.M., The Constitution of Canada, Toronto, 1922, 166-81.

5. CF. SIMEON, R., Federal Provincial Diplomacy, 'Toronto, 1972; pour le discours de M. Dunkin, voir WAITE, P.B., ed., The Confederation Debates in the Province of Canada 1865 Toronto, 1963; voir les discours de Messieurs MacDonald, Galt, et Brown pour la position centralisatrice.

6. Cf. RUSSELL, P., ed., Leading Constitutional Decisions, Toronto, 1973, I et II; MALLORY, J.R., The Structure of the Canadian Government, Toronto, 1971, 26s., 228-48; BLACK: 4 chap.; STANLEY, G.P.G., A Short History of the Canadian Constitution, Toronto, 1969, chap. 5, 6 . 
Depuis la crise, cependant, le centralisme originel de la Constitution s'est réaffirmé. L'État central s'est emparé des principales fonctions en matière de bien-être, est entré dans des domaines provinciaux (comme les affaires urbaines, l'éducation, la santé), et a étendu ses pouvoirs culturels. Ses subventions aux provinces ont eu pour effet de forcer les gouvernements provinciaux à changer leurs priorités. Tout ceci a eu pour résultat que la responsabilité démocratique de chaque gouvernement s'est trouvée minée par la compétition intergouvernementale, ainsi que par la confusion et la complexité croissantes de la Constitution. C'est cette période de centralisation qui est à l'origine des exigences d'un Québec réclamant des pouvoirs gouvernementaux accrus, allant jusqu'à l'indépendance. Les propositions du parti québécois en vue d'une nouvelle entente Québec/Canada sont encore plus étatistes. Elles créeraient deux États centraux puissants. L'association entre les États serait elle-même étatiste: elle serait faite par traité. Et, finalement le parti québécois propose un ensemble de mécanismes intergouvernementaux d'association politique, qui ne seraient pas soumis au suffrage universel ${ }^{7}$.

En même temps, les provinces sont devenues aussi plus riches et plus puissantes, en raison de changements sociaux et économiques. Tout cela a provoqué des demandes pour une réforme de la Constitution, mais elles n'ont cependant pas encore donné lieu à une discussion prolongée sur une nouvelle division des pouvoirs. Les propositions Pépin-Robarts et Ryan sont remarquables en ce qu'elles veulent faire revivre une Chambre haute fédérale dont les membres sont nommés, mais une telle mesure doterait les provinces d'un mécanisme étatiste leur permettant d'influencer les décisions de l'État central. Ces deux études clarifient cependant la division des pouvoirs, la rendent plus flexible, et accordent plus de pouvoir aux provinces.

En 1980, les gouvernements provinciaux et le gouvernement fédéral ont discuté d'une réforme éventuelle de la division des pouvoirs, mais seulement pendant une période relativement courte de quelques mois. Le débat a atteint son point culminant dans le cul-de-sac de la Conférence constitutionnelle de septembre, et dans la décision de $M$. Trudeau sur le rapatriement

7. Cf. SIMEON: 213s. SMILEY, D.V., Canada in Questions, 2e édition, Toronto, 1976, 74s., 202; et Le Gouvernement du Québec, La Nouvelle Entente Québec-Canada, Québec, 1979, 64s. 
unilatéral de la Constitution. Même si les provinces cherchaient clairement à protéger et à accroître leurs pouvoirs étatiques, il y avait néanmoins des signes qu'on aurait pu parvenir à un accord sur la question centrale du contrôle des ressources naturelles par les provinces. Un tel accord aurait pu rendre possible une certaine flexibilité concernant d'autres questions, comme celles par exemple des droits linguistiques et de la formule d'amendement. L'affirmation, par les provinces, de la souveraineté et du majoritarisme parlementaires c'est-à-dire étatiques contre la garantie des droits civils et des droits des minorités, a renforcé l'idée que l'étatisme provincial et la tradition de «representation by population» sont encore très forts au Canada. Un consensus canadien est encore à faire concernant l'importance des droits collectifs des minorités dans notre démocratie fédérale.

La décision subséquente et unilatérale de $M$. Trudeau, c'està-dire rapatrier la Constitution et imposer une formule d'amendement, un principe d'égalisation régionale et une charte des droits civils et linguistiques, doit elle-même être criitiquée pour les conceptions du nationalisme, du libéralisme et du fédéralisme qu'elle véhicule. Elle réaffirme la tradition canadienne libérale du nationalisme constitutionnel vis-à-vis de la Grande-Bretagne, mais contredit l'opposition libérale au nationalisme économique vis-à-vis des États-Unis et au nationalisme culturel à l'intérieur du Canada. La proposition d'Ottawa ne reconnait pas les droits culturels et linguistiques des Amérindiens et des minorités canadiennes-françaises comme telles, en Ontario, au Manitoba et au Nouveau-Brunswick, et elle pourrait saper les gains récents de la collectivité francophone du Québec. Les sections portant sur les droits civils sont affaiblies par une formulation vague et par la généralité des exceptions. Beaucoup de Canadiens, fatigués des querelles intergouvernementales sur la Constitution, accueillent favorablement l'esprit de décision que traduit l'initiative de Trudeau. L'unilatéralisme rigide de cette initiative mine cependant la crédibilité de l'engagement d'Ottawa en faveur d'un fédéralisme démocratique.

En dépit de leur valeur, ces propositions sont encore reliées à la question des pouvoirs des gouvernements beaucoup plus qu'à la démocratie. Tant que la question de la réforme constitutionnelle demeurera confinée aux limites de ce contexte étatiste, elle mè- 
nera inéluctablement à des impasses, car toute discussion entre gouvernements sur le partage de leurs pouvoirs respectifs place ces mêmes gouvernements dans une inévitable situation de conflit d'intérêts: chacun doit défendre ses propres intérêts et protéger ses propres pouvoirs. Ceci a pour conséquence de rendre pratiquement impossible toute discussion franche et ouverte sur ce que serait la meilleure division des pouvoirs. La triste réalité dans tout cela, c'est qu'aucun gouvernement ne renoncera de lui-même à ses pouvoirs.

La morale de ce réalisme, c'est que seul un processus démocratique, impliquant les communautés et les citoyens dans l'intérêt desquels les gouvernements exercent le pouvoir, sera à la fois prêt à redistribuer les pouvoirs gouvernementaux dans la fédération et à redéfinir les structures de cette dernièren et capable de le faire; car un tel processus se déroulera dans l'intérêt de ces communautés et de ces citoyens, et en fonction de leur bien commun.

Examinons maintenant la coïncidence des intérêts particuliers et du bien commun dans cette alternative de fédéralisme démocratique.

\section{VERS UN FÉDÉRALISME DÉMOCRATIQUE}

Il y a des signes d'un renouvellement démocratique du fédéralisme canadien: entre autres un consensus de plus en plus grand pour que le Canada soit un pays bilingue et pour que toute nouvelle constitution, - ou l'indépendance du Québec, — soit ratifiée par le peuple. La réforme du Sénat, telle que conçue par Trudeau, permettrait aux Législatures et aux gouvernements provinciaux d'y être représentés. Le droit du Québec à l'autodétermination est implicitement admis dans le refus généralisé d'employer la force contre tout choix démocratique en vue de l'indépendance. Les propositions concernant les chartes de droits et la représentation proportionnelle, bien que n'étant pas expressément fédérales, témoignent de sentiments démocratiques. Les référendums nationaux sont cependant inadéquats d'un point de vue fédéraliste, dans la mesure où la forme même du scrutin homogéniéise la diversité du sentiment public réel dans la so- 
ciété. Dans de telles stratégies se cache un principe de démocratie, non fédéral, individualiste et majoritariste, ennemi de la protection des droits collectifs des minorités.

Depuis les rébellions des années 1830 jusqu'aux années 1860 , les Canadiens ont demandé une autonomie politique et économique plus grande par rapport au contrôle impérial. Après 1867 , les rébellions de la rivière Rouge, le mécontentement du Québec et des Maritimes, le radicalisme de l'Ouest, et les débats courants sur l'exploitation régionale, l'assimilation culturelle et les excès du pouvoir central canadien, furent tous fédéraux quant à la forme. Les protestations des groupes sociaux, ethniques et culturels se manifestèrent par une résistance régionale aux pouvoirs centraux et par des demandes d'autonomie provinciale ${ }^{8}$.

Les mouvements prônant l'autonomie provinciale en Ontario, au Québec et en Alberta, par exemple, ont été le reflet de luttes démocratiques de la part des groupes déjà mentionnés contre l'exploitation et/ou l'assimilation. Les régimes provinciaux ont été dominés par des partis progressistes (par exemple) les libéraux de l'Ontario, les Fermiers Unis, le CCF/NDP, les rouges du Québec, le $\mathrm{PQ}$ ). Les droits des provinces impliquent le principe libéral de la limitation du pouvoir de l'État central, et donc de l'accroissement de l'autonomie locale. L'interprétation provincialiste de l'Union comme pacte fédéral entre des gouvernements provinciaux coloniaux, même si elle n'a aucun fondement historique, est en fait une théorie plus fédérale que la forme actuelle de l'Union, car le terme "fédéral" vient d'un mot latin qui signifie "pacte" ${ }^{9}$.

Ceci est encore plus vrai des théories d'un pacte culturel, d'une entente entre anglophones et francophones. Bien que moins précises, elles reposent sur des fondements historiques solides, impliquant les ententes non écrites sous-jacentes aux négociations qui ont mené à l'Union de 1867. Leur importance est inestimable, étant donné que les exigences actuelles en vue d'un fédéralisme renouvelé constituent une poussée vers un pacte social

8. Sur l'inégalité régionale, voir The (Pépin-Robarts) Task Force on Canadian Unity Report, $A$ Future Together. Ottawa, 1979, 6е chap.; SMILEY: 192s.; BERCUSON, D., ed., Canada and the Burden of Unity, University of Toronto, 1977.

9. Cf. STANLEY; BLACK: $5 e$ chap. et VERNON, R., ed., The Principle of Federation by P. $-J$. Proudbon, University of Toronto, 1979, 7e chap. 
explicite entre les gouvernements membres et les communautés du Canada. Une réflexion sur les implications d'un pacte social au sein d'une entente biculturelle pourrait mettre en lumière une politique de la communauté inhérente à un fédéralisme démocratique.

Tout système politique doit être représentatif, dans sa forme, de la diversité sociale de sa "matière», c'est-à-dire de la société qu'il gouverne. Dans la mesure où l'État est basé sur l'intégrité territoriale et constitue une unité politique indifférente, quant à sa forme, à l'histoire et à la complexité de la société qu'il gouverne, sa forme unitaire est moins adaptée aux sociétés socialement complexes que sa forme fédérale. L'État-nation constitue une alternative collectiviste à un État purement territorial et à un système fédéral. En pareil cas au moins, la composition interne et la continuité historique d'une société représentent des critères de légitimité de cette forme de gouvernement. L'homogénéité de la culture d'une société requiert donc que cette société soit gouvernée par son propre État national. Ce principe culturel nationaliste est ce qui a mené aux exigences des anglophones pour «un seul Canada», ainsi qu'aux exigences du Québec en vue de l'obtention du statut complet de nation. Ces exigences sont antifédérales, dans l'un et l'autre cas. Elles tendent à transposer le biculturalisme dans une théorie des deux nationi, et, par conséquent, de deux États, que ceux-ci soient associés dans le cadre d'une fédération ou séparés et pleinement souverains. Le fédéralisme démocratique rejette donc à la fois l'étatisme territorial et national, et, corollairement, l'étatisme associé.

Le Canada se dirige ainsi vers un nouveau pacte fédéral. Ceci soulève certaines questions sur la nature du pacte lui-même en tant que forme d'association politique, sur les partenaires impliqués, et sur les arrangements qui peuvent être faits dans le cadre d'un tel pacte. Premièrement, il y a déjà une tradition voulant que le pacte fédéral soit une association volontaire entre partenaires négociant sur un pied d'égalité. J'appellerai «pacte social» une telle entente volontaire menant à une fédération démocratique sous une seule constitution. L'histoire et la pratique du fédéralisme canadien suggèrent que les partenaires du pacte soient des collectivités - provinces, régions, cultures, villes, etc. 
Le pacte social ressemble à un contrat social, mais les deux notions sont cependant distinctes. Les contrats sociaux lient des individus dans des relations prépolitiques et présociales, tandis que les pactes lient des sociétés politiques existantes. Dans la théorie fédéraliste canadienne, les partenaires du pacte sont des communautés. De plus, les contrats sociaux ne sont pas par définition fédéraux, tandis que le pacte social est par essence un pacte fédéral. La notion de pacte social differe de celle de contrat social à d'autres points de vue encore. S'il n'y a jamais eu de contrat social, il y a cependant eu des pactes sociaux. Il y a eu ainsi des confédérations réelles qui ont existé (par exemple chez les Iroquois).

Le fédéralisme canadien doit également rejeter l'individualisme atomiste abstrait qu'implique la théorie du contrat. Un tel individualisme est abstrait parce qu'il postule l'immédiateté des individus contractants dans leurs relations mutuelles et dans leur relation à l'État. Une telle immédiateté politique, en plus d'être inappropriée à la situation canadienne dans laquelle representation by population est un slogan de division, et l'individualisme radical, une idéologie étrangère, constitue aussi un mythe ${ }^{10}$. Il s'agit en fait d'une forme séculière du protestantisme. Dans un tel contexte, la liberté subjective de l'individu est définie par une opposition simpliste à la domination objective des institutions sociales autoritaires, telles que la famille, l'Église et l'État. Une indépendance, ou non-dépendance radicale, de la volonté des autres est considérée comme identique à la liberté. Mais personne ne peut être complètement indépendant par rapport aux autres, ni se suffire à soi-même.

Finalement, la conséquence de l'individualisme ne peut être que l'asservissement à l'autorité rel igieuse, politique, et économique - autrement dit, à la Bible, à l'État et à la corporation; car les individus ne peuvent s'unir en vue d'accroître leur pouvoir contre l'autorité sans perdre par le fait même leur liberté.

L'individualisme politique est également atomiste en ce qu'il réduit chaque être humain à une unité parfaitement sembla-

10. Au sujet de l'individualisme, voir MILLER, M., ed., Peter Kropotkin: Selected Writings on Anarchism and Revolution, Cambridge, 1972, 293-307; LUKES, S., Individualism, Oxford, 1978; et HOROWITZ, G., Canadian Labour and Politics, Toronto, 1968: le chap. I. 
ble aux autres du même type, du point de vue de son adhésion au contrat social. À partir, de là, on passe aisément au troupeau ou à la masse. Un tel individualisme est finalement subjectiviste: la liberté s'identifie à la satisfaction instantanée et totale des désirs de l'individu, dans un quelconque état de nature mythique. Ceci est à la fois le point le plus bas de l'éthique et le contraire du fondement de la démocratie. Il s'agit encore ici de protestantisme sécularisé, mais seulement en rapport avec le mythe du péché originel et la corruption de la nature humaine.

La théorie individualiste suggère des formes d'association purement contractuelles, du genre des relations contractuelles ou commerciales entre individus distincts et entre communautés d'intérêts purement passives. De telles formes sont articulées dans des institutions démocratiques de masse ou de marché, comme les partis électoraux, les groupes d'intérêts, et d'autres semblables.

Le pacte social et les théories démocratiques, par contre, sont plus près à la fois des idéaux et de la réalité, car ils assument l'individuel concret dans la mutualité sociale. Ils critiquent seulement les relations sociales qui font du tort à l'individualité, à la liberté et à la démocratie. La théorie du pacte considère que l'individualité ne se contente pas d'exister: elle se développe. Ce développement nécessite du temps, du savoir et un processus de socialisation. L'immédiateté n'est donc ni désirable ni possible. On doit plutôt chercher les lois fondamentales de la médiation, en vue de trouver des modalités compatibles avec les principes démocratiques et fédéralistes, ou individualistes et collectivistes.

La théorie du pacte social postule donc que l'homme est un animal social, et que la sociabilité est naturelle, donc essentielle au développement de la liberté et de l'identité de la personne, ainsi qu'à l'égalité et à la justice sociales. Dire que la sociabilité est naturelle n'implique pas qu'elle soit antidémocratique. La notion de nature s'oppose à celle de convention, mais pas à celle de démocratie. De plus, les sociétés, qu'elles soient autoritaires ou démocratiques, individualistes ou collectivistes, sont toujours conventionnelles, même lorsque les conventions élaborent ce qui est naturel aux humains, comme, par exemple, le fait de vivre en société. 
Il s'ensuit que les partenaires du pacte fédéral démocratique devraient être des communautés intrinsèquement démocratiques, que j'appellerai ici "communautés politiques fédérales». Les critères de démocratie mentionnés ci-dessus peuvent en pratique être réalisés à des degrés divers. Mais certaines des communautés considérées ici comme des partenaires potentiels dans un pacte fédéral canadien sont soit des associations "naturelles", soit des entités qui ne sont pas en elles-mêmes très démocratiques, comme par exemple des cultures et des villes. Dans la mesure où toutes les formes humaines d'association sont jusqu'à un certain point conventionnelles et constituent des produits de l'artifice humain, la première objection disparaît. Je répondrait à la seconde objection que le processus de développement d'un pacte social politiserait par lui-même de nombreuses communautés. Cela s'est produit déjà dans la politique culturelle du Canada ${ }^{11}$. La politisation démocratique des communautés du Canada n'est donc pas impossible. Elle demeure cependant une chose délicate et difficile.

Ce qui est en question ici, c'est le type de relations sociales qui prévalent dans toute collectivité: les relations coercitives, manipulatrices, secrètes, autoritaires, de même que les relations de compétition et d'exploitation, doivent être éliminées ou réduites au minimum. Les relations coopératives, volontaires, ouvertes, mutuelles et égalitaires doivent être maximisées. Le pouvoir et les biens doivent être partagés au lieu d'être concentrés. De cette manière, l'opposition simpliste entre individu et communauté peut être amenée à disparaître.

Le but de cette digression est de montrer que le point de départ de la théorie du pacte social, à savoir que les partenaires d'une fédération doivent être des communautés démocratiques, n'est ni contradictoire ni irréaliste, ou, du moins, qu'il est plus cohérent et plus réaliste que la théorie du contrat social. On doit naturellement rejeter le concept abstrait de la communauté comme n'importe quel ensemble d'êtres humains, disparates bien

11. Cf. KWAVNICK, D., ed., The Tremblay Report, McClelland, Toronto, 1963: chap. 6, 7; DION, L., Québec: The Unfinished Revolution, Montréal, 1976; BERGER, JUDGE THOMAS, Northern Frontier, Nortbern Homeland: Report of the Mackenzie Valley Pipeline Inquiry, vol. I, Ottawa, 1977, 91 s., 194s.; MANUEL, G., ET POSLUNS, M., The Fourth World, Galt, 1974; et, pour une exposition générale, voir SMITH, A., Theories of Nationalism, London, 1972. 
qu'associés, qui sont momentanément rassemblés ou qui partagent une caractéristique quelconque. Cette distorsion individualiste est inacceptable. On admet communément que le terme de «communauté» est ambigu et qu'il est de ceux qui présentent certaines difficultés pour la théorie démocratique. Certains critères relatifs à la notion de communauté sont par conséquent requis. Je voudrais en proposer trois, en précisant qu'ils n'entraînent pas par eux-mêmes des structures politiques démocratiques (ou autoritaires). Ces trois critères sont la vie sociale commune, la territorialité, et la temporalité. Ces trois éléments forment ensemble de simples critères de ce qui constitue une communauté et, par conséquent, un partenaire potentiel dans un pacte social.

La vie sociale commune fait partie de la notion courante de communauté, définie comme un groupe de gens qui vivent ensemble dans un même lieu pendant une période de temps importante, et qui profitent en commun d'un système de communications, de certaines valeurs, d'une économie, d'un système politique, etc. Ce ne sont pas là, à mon avis, des caractéristiques passives: vivre ensemble, c'est parler, travailler, discuter, etc., les uns avec les autres (ou les uns contre les autres). D'une part, ce concept tend vers la notion d'une culture commune; d'autre part, il suggère un consensus ou un système commun de croyances. Il peut également être étendu à un groupe comportant des gens de cultures et de croyances différentes, et qui ont vécu ensemble pendant une période de temps importante. Ce concept n’implique finalement ni la paix générale ni l'harmonie. Les conflits de classes, tout comme les conflits religieux, politiques, culturels, etc., ne se retrouvent pas que dans la communauté, mais ils peuvent parfois la caractériser.

Le territoire est fondamental pour toute communauté humaine. Mais cette territorialité ne correspond à aucun instinct agressif ou prédateur. L'intégrité territoriale, par extension, est fondamentale pour la souveraineté politique, que ce soit dans un État ou une démocratie. Innis a noté que le contrôle de l'espace est essentiel pour toute organisation politique. Et, comme Heidegger l'a écrit, l'existence humaine est essentiellement spatiale; l'homme est da-sein, être là ${ }^{12}$. C'est tout à fait vrai en pratique: les

12. Voir HEIDEGGER, M., Being and Time, New York, 1963, sections 23, 24, 73 à 77; INNIS, H.A., The Bias of Communications, Toronto, 1952; et son Empire and Communications, Toronto, 1954. 
Canadiens de toutes les cultures s'identifient très fortement à la terre canadienne.

La politique culturelle montre l'importance de la tradition et de l'histoire, en résumé du temps pour la communauté. Ceci ne revient pas à dire que vivre dans une communauté implique qu'on est traditionaliste; cela implique seulement que la vie sociale doit se maintenir au cours de plusieurs générations. La continuité entre le passé et le futur est essentielle pour toute conscience et toute existence individuelle et sociale. Et, comme Innis I'a fait ressortir, la continuité historique constitue un autre trait essentiel de l'organisation politique. La courte histoire du Canada, et les taux élevés d'immigration et d'émigration qui caractérisent notre pays, représentent des objections importantes contre cette dernière considération. Notre mobilité géographique considérable ajoute donc une difficul té supplémentaire à la théorie du pacte des communautés. Il reste encore malgré tout plusieurs communautés dans le pays; mais ce dernier n'est pas lui-même une communauté simple et unique.

L'une des bases du fédéralisme est, en fait, la complexité de la société qu'il faut gouverner. Une société aussi complexe que la nôtre dans ses dimensions régionales et culturelles, et qui occupe un si vaste territoire, constitue un cas classique favorisant un système fédéral beaucoup plus qu'un État unitaire. Il s'agit de donner un appui et des moyens d'expression adéquats aux régions et aux cultures incluses dans la fédération, tout en développant en même temps un système de gouvernement démocratique et stable. Dans la mesure où l'on conserve un système étatiste à l'intérieur d'une fédération, on contredit à mon avis les exigences ci-dessus mentionnées, voulant que la complexité sociale soit représentée et que les diverses collectivités soient assurées de canaux d'expression. Donc les arguments qui s'appuient sur la complexité sociale de la base pour montrer le besoin d'un système fédéral soutiennent également l'idée que ce système doit être démocratique, et non pas étatiste.

Mais quelles communautés devraient être des partenaires dans un pacte social qui constituerait la base d'une reconstruction démocratique de la fédération canadienne? Les États sont éliminés. La politique culturelle du Canada suggère que les cultures 
constituent un type de partenaire collectif dans le pacte. La croissance du nationalisme canadien-français et québécois, et la résurgence culturelle quasi nationale des peuples amérindien et inuit, constituent des formes culturelles de résistance démocratique à un fédéralisme culturellement assimilateur; car il s'agit, en fait, de protestations contre le manque de garanties authentiquement fédérales pour les communautés culturelles impliquées. Les droits linguistiques se rapportent donc à des groupes, et non pas simplement à des individus. Ce que tous ces groupes recherchent, ce sont plutôt des formes variées de contrôle démocratique de leur vie culturelle collective ${ }^{13}$. Les tribus amérindiennes recherchent des formes de pouvoir aux niveaux municipal et provincial, incluant la propriété commune du sol, et une représentation garantie au Parlement central. Les Canadiens français à l'extérieur du Québec cherchent à obtenir des garanties relatives aux droits des minorités, concernant tout particulièrement les services et l'éducation dans leur propre langue; c'est ce que recherchent également les anglophones du Québec. Les Québécois, naturellement, cherchent à parvenir à un certain niveau de gouvernement national. Les groupes d'immigrants essaient de préserver certaines de leurs habitudes et d'obtenir la garantie que leurs enfants apprendront à l'école leur langue maternelle, tout en acceptant la (les) culture(s) canadienne(s) dominante(s).

Les cultures canadiennes, selon moi, ce sont la culture anglaise et la culture française. L'italien n'est pas une langue canadienne; et les peuples aborigènes préexistaient par définition à la création d'États modernes européens dans les colonies britanniques et françaises. Les luttes de ces dernières ont été surimposées sur le substrat des sociétés amérindiennes, qu'elles ont menées ensuite à leur déclin. Les mécanismes devant servir à l'expression démocratique et à la protection des cultures mentionnées ne sont pas tous décentralisés. C'est pourquoi les francophones et les Amérindiens cherchent une protection constitutionnelle et une représentation au niveau des institutions fédérales. Enfin, ces groupes ne constituent pas tous des communautés véritables. Le degré de dispersion des Canadiens français à l'extérieur du Québec, des Amérindiens dans les villes, et des immigrants à l'exté-

13. Voir The Tremblay Report, 1re parrie; MANUEL et POSLUNS, 9e chap; ; et BERGER, 8. chap. 
rieur de certaines villes ou de certains quartiers, est tel que l'on doit souvent parler de familles et d'individus isolés plutôt que de communautés. Mais, quel que soit le lieu où existent des communautés tribales, françaises, anglaises ou immigrantes, les mécanismes mentionnés ci-dessus sont appropriés. Ils s'appliquent aux «diasporas culturelles» au moins par extension, et dans la mesure du possible. On admet généralement que le trait distinctif de la société canadienne consiste dans le fait qu'elle est menacée par la pénétration économique et culturelle américaine. Certains diront que la meilleure solution à ce problème réside dans un gouvernement central ou national plus puissant.

Ni les familles ni les sexes ne peuvent être considérés comme des communautés politiques fédérales dans le contexte d'un nouveau pacte social, ces entités étant trop dispersées ou d'une taille trop petite. Les groupes religieux, moins dispersés, présentent souvent une certaine cohésion de même qu'une certaine continuité, et partagent jusqu'à un certain point une vie sociale commune. Le consensus séculier dans la société moderne milite cependant contre le fait que l'on reconnaisse les religions comme partenaires possibles dans le pacte. Il n'en serait pas ainsi naturellement dans une société plus religieuse, dans laquelle un telle reconnaissance ne serait pas nécessairement vue comme antidémocratique. Dans la mesure où les classes économiques forment des communautés d'intérêts unifiées, conscientes d'elles-mêmes, durables et cohérentes, elles pourraient, semble-t-il, être reconnues éventuellement comme communautés politiques fédérales. Mais je crains que les critères de la communauté ne s'appliquent d'une manière inégale entre les classes riches, moyennes et pauvres. De plus, les revendications d'ordre économique peuvent déjà s'articuler, quoique d'une manière imparfaite, au sein de la politique non fédérale habituelle. Dans la mesure cependant où les classes moyennes et inférieures développeraient une cohésion et une force plus grandes, leur reconnaissance comme communautés politiques fédérales deviendrait plus problable et nécessaire.

Dans le cas des régions et des villes, nous trouvons des démarcations territoriales relativement claires entre des communautés qui possèdent un degré significatif de vie sociale commune et de continuité. Elles peuvent et doivent donc être reconnues comme communautés politiques fédérales. Qu'elles ne soient pas 
ainsi reconnues constitue un réquisitoire contre la présente Constitution. Les communautés culturelles d'Amérindiens, de Canadiens français et de Canadiens anglais sont considérées comme des communautés politiques fédérales pour des raisons similaires; mais alors que ces dernières sont surtout définies par leur continuité historique, les régions, les villes, etc., forment des communautés sur la base du territoire. L'espace et le temps constituent donc les points de référence permettant de définir les communautés politiques qui devraient être membres et partenaires dans un nouveau pacte fédéral.

La proposition de pacte social est donc une manière de faire du Canada une communauté de communautés ${ }^{14}$. Elle reconnaît en même temps que le Canada d'aujourd'hui n'est pas une communauté de communautés. Cela prendra beaucoup de temps, mais le processus de renouvellement démocratique du fédéralisme canadien par la négociation d'un pacte social pourra lui-même aider à la formation des communautés politiques essentielles à ce pacte. C'est le projet de pacte social qui encourage les germes déjà existants à croître en une plante fédérale vigoureuse. Ces germes, selon moi, sont déjà en train de s'épanouir dans les cultures, les régions et les cités du Canada.

Cet argument est donc circulaire, mais les cercles ne sont pas tous vicieux, ou purement logiques. On a plutôt affaire à un cercle pratique de rétroactions lorsqu'on travaille à renouveler nos arrangements politiques en essayant de définir les communautés qui seront partenaires dans un pacte social nouveau, et en essayant de définir ce pacte lui-même. La démocratisation du Québec, qui est la société la plus engagée dans la recherche d'une nouvelle forme d'association, est un indicateur de la validité de ce projet. Cela impliquera, premièrement, un processus dans lequel on mettra de côté les gouvernements officiels parce qu'ils ne sont pas des organismes appropriés pour faire le renouvellement du fédéralisme. Le fait qu'ils se trouvent en conflit d'intérèts dès qu'on leur demande de redistribuer leurs pouvoirs et de démocratiser la

14. Par contraste aux orientations ou étatistes ou individualistes dans: Le rapport du Parti libéral du Québec, La Nouvelle Fédération canadienne. The Pépin-Robarts Report; et les deux propos du gouvernement du Canada, Le projet d'une loi sur la réforme constitutionnelle (1978), articles 13 à 22 , et La loi constitutionnelle de 1980, articles 16-23. 
Constitution devrait dissiper immédiatement nos illusions sur le sujet.

Le système par lequel une société aussi complexe que la nôtre devrait et pourrait être gouvernée doit plutôt être développé au moyen d'un pacte mutuel volontaire entre les communautés politiques qui constituent la société canadienne. Je n'exclurais pas de ce processus les gouvernements et les partis de l'actuelle fédération canadienne, mais ils ne devraient pas le contrôler, pour les raisons mentionnées ci-dessus. Les communautés régionales, urbaines et culturelles qui composent la société canadienne doivent en arriver à être reconnues pleinement et politiquement en tant que membres de la fédération. Ceci ne peut être accompli qu'en impliquant ces communautés comme telles dans le processus de réforme de la fédération, c'est-à-dire dans une conférence constitutionnelle où elles seraient adéquatement représentées. Finalement, les accords qui y seraient conclus devraient être ratifiés dans des référendums populaires, car la souveraineté populaire doit remplacer la souveraineté de l'État. Mais de tels scrutins devraient être tenus par les communautés politiques membres de la fédération, au lieu de se dérouler à l'échelle du pays, car la fédération n'est pas basée sur un contrat social entre individus, mais bien plutôt sur un pacte social entre communautés.

En guise de remarque finale, je dirai ceci: définir la forme du pacte et les critères du choix des partenaires n'est pas la même chose que décider de la structure que ces partenaires créeront par leur pacte. Mais cette structure ne pourrait être incompatible avec les principes démocratiques sous-jacents au pacte. Un pacte social, dans la mesure où il est fidèle à l'esprit qui lui est sousjacent, ne peut pas produire un État. même «fédéral». Mais cela ne veut pas dire qu'il doive produire un système politique fortement décentralisé, à deux (ou plusieurs) niveaux. En fait, le pacte pourrait entraîner la création d'un niveau de gouvernement unique, dans la mesure où une telle instance gouvernementale serait démocratique, et où ses structures pourraient être contrôlées démocratiquement par les communautés partenaires; mais ça ne veut pas dire que ces communautés seraient elles-mêmes les gouvernements ou les niveaux de gouvernements dans la nouvelle constitution. C'est à elles d'en décider, au niveau de leur pacte. Des garanties claires portant sur les divers droits, intérêts, etc., 
doivent faire partie des nouveaux accords. Étant donné que la souveraineté populaire et l'autonomie de la communauté constituent l'essence du pacte social, il faudrait qu'elles soient les principes effectifs de la nouvelle fédération.

Département de philosophie Université de Sudbury 